\title{
Risk of cancers of the oesophagus and stomach by histology or subsite in patients hospitalised for pernicious anaemia
}

\section{W Ye, O Nyrén}

See end of article for authors' affiliations

\section{................... \\ Correspondence to:} Dr W Ye, Department of Medical Epidemiology, Karolinska Institutet, Box 281, SE171 77,

Stockholm, Sweden

Weimin.Ye@mep.ki.se

Accepted for publication 7 February 2003
Background: Although pernicious anaemia is an established risk factor for stomach cancer, data by anatomical subsite are not available. Moreover, a previous suggestion of a link to increased risk of oesophageal cancer needs further exploration.

Methods: We followed 21265 patients hospitalised for pernicious anaemia in Sweden from 1965 to 1999 for an average of 7.1 years. Standardised incidence ratio (SIR) adjusted for sex, age, and calendar year was used to estimate relative risk, using the Swedish nationwide cancer incidence rates as reference.

Results: Significant excess risks for squamous cell carcinoma of the oesophagus, and stomach cancer distal to the cardia, were observed in pernicious anaemia patients (SIR 3.3 (95\% confidence interval (CI) 2.4-4.4); SIR 2.4 (95\% CI 2.1-2.7), respectively). The excess risks increased with increasing follow up duration. Among distal stomach cancers, the most conspicuous excess risk was for carcinoid tumours (SIR $26.4(95 \% \mathrm{Cl} 14.8-43.5))$. Compared with the general population, no significant increased risk was observed for adenocarcinoma of the oesophagus (SIR $1.7(95 \% \mathrm{Cl} 0.7-3.4)$ ) or gastric cardia (SIR $1.2(95 \% \mathrm{Cl} 0.6-2.0))$.

Conclusions: Achlorhydria following type A atrophic gastritis is associated with an elevated risk of adenocarcinoma of the non-cardia stomach, and surprisingly, with a risk of oesophageal squamous cell carcinoma. In contrast, no significant association, either positive or negative, was found with oesophageal or cardia adeoncarcinoma. The mechanism for the observed increased risk of oesophageal squamous cell carcinoma warrants further study.
$P$ ernicious anaemia is the end stage of type A atrophic gastritis influencing the fundus and body of the stomach. ${ }^{1}$ The lesion may persist for 20-30 years before a clinical diagnosis of pernicious anaemia is made. An increased risk of stomach cancer among pernicious anaemia patients has been observed in several cohort studies. ${ }^{2-5}$ There is also a suggested excess of oesophageal cancer among these patients, ${ }^{3}$ but this observation needs to be verified in a larger study. Accumulating data indicate that the aetiology of oesophageal adenocarcinoma is different from that of oesophageal squamous cell carcinoma, and gastric cardia adenocarcinoma has risk factors that are distinct from those for non-cardia stomach adenocarcinoma. ${ }^{6}$ Gastro-oesophageal reflux has been reported to be an important risk factor for oesophageal adenocarcinoma and, to a lesser extent, for cardia cancer. ${ }^{7-9}$ However, it remains unclear which substance in the refluxate plays a crucial role in carcinogenesis of these cancers. ${ }^{10}$ If gastric acid is carcinogenic to the mucosa of the gastro-oesophageal junction, achlorhydria in pernicious anaemia should be followed by a decreased risk of these cancers. The observed inverse association between Helicobacter pylori infection and risk of oesophageal adenocarcinoma has been tentatively attributed to $H$ pylori induced atrophic gastritis and hypochlorhydria. ${ }^{11}$ The risk of adenocarcinoma in the gastro-oesophageal junction in patients with long term achlorhydria has however not been investigated in previous studies.

We conducted a register based retrospective cohort study to estimate the risk of oesophageal cancer by histology and risk of stomach cancer by anatomical subsite, among patients hospitalised for pernicious anaemia.

\section{METHODS}

The record linkage methodology used here has been described in detail elsewhere. ${ }^{12}$ The Swedish Inpatient Register contains data on essentially all inhospital care in the population covered by the registration. It started in 1964-65 and has covered all of Sweden since 1987. We identified a total of 31027 unique national registration numbers (unique identifiers assigned to all Swedish residents) which were registered at least once with a discharge diagnosis of pernicious anaemia (1965-68, Swedish revised ICD-7=290.00, 290.10; 1969-1986, revised ICD-8 $=281.00,281.09 ; 1987-96$, revised ICD-9=281A; 1997-99, revised ICD-10=D159, D510). This cohort was linked to the nationwide Register of Causes of Death to identify the date of death, and to the Emigration Register to identify the date of emigration. Cancer prevalence at entry and incidence during follow up were ascertained in the Swedish Cancer Register, founded in 1958 and estimated to be 98\% complete. ${ }^{13}$ Through additional linkage to the Total Population Register, we excluded 181 records with erroneous national registration numbers, not identifiable in any of the aforementioned registers. We also excluded 3745 patients with prevalent cancers at the time of entry as we included only first primary cancers in this study. To minimise the impact of possible selection bias, we excluded the first year of observation after the index hospitalisation in all analyses. We thus excluded 3907 patients who were followed for less than one year. We excluded a further 1626 patients who died during the

Abbreviations: SIR, standardised incidence ratio. 
Table 1 Characteristics of the patients hospitalised for pernicious anaemia: Sweden, 1965-1999

\begin{tabular}{lrl}
\hline Characteristic & & \\
\hline No of patients & 21265 & \\
Distribution by sex (\%) & 8442 & $(39.7)$ \\
$\quad$ Male & 12823 & $(60.3)$ \\
Female & 74.3 & 7.1 \\
Average age at entry (y) & 161672 & \\
Average duration of follow up (y) * & 507 & $(2.4)$ \\
Total person years accumulated & 82 & $(0.4)$ \\
Alcoholism (\%) & 421 & $(2.0)$ \\
Alcoholic liver cirrhosis (\%) & 911 & $(4.3)$ \\
Pancreatitis (\%) & & \\
Alcoholism, alcoholic liver cirrhosis, or & & \\
pancreatitis & &
\end{tabular}

index hospitalisation, 77 patients with inconsistencies uncovered during record linkages, and 174 patients who had gastric resection surgery before entry. As childhood pernicious anaemia is more likely to be caused by genetic disorders that result in lack of intrinsic factor or defective intrinsic factor than atrophic gastritis, ${ }^{1} 52$ patients who had a diagnosis of pernicious anaemia before the age of 20 years were also excluded. Finally, a total of 21265 patients, 8442 men and 12823 women, remained for further analysis.

Follow up began one year after the discharge date of the index hospitalisation for pernicious anaemia until cancer diagnosis, emigration, death, or end of the observation period (31 December 2000), whichever came first. The Cancer Register recorded neoplasms using the ICD-7 classification throughout the entire study period. The histological type of each recorded tumour was also identified by a histology code. As a separate code for gastric cancer in the cardia was introduced first in 1969, cases and person years in the stomach cancer analyses were accumulated from 1970, one year after the coding change, to ensure quality of data. To avoid possible ascertainment bias associated with differential autopsy rates between pernicious anaemia patients and the general population, we did not include cancers found incidentally at autopsy. Relative risk of cancer in the cohort was estimated by the standardised incidence ratio (SIR) - that is, the ratio of the observed number of cancers to that expected-derived from the Swedish general population without a reported cancer. The $95 \%$ confidence interval (CI) of the SIR was calculated by the exact method on the assumption that the observed number of cases follows a Poisson distribution. ${ }^{14}$

To control for the confounding effect of alcohol intake, we identified alcohol related comorbidities-that is, alcoholism (Swedish revised ICD-7 $=307,322$; ICD-8 $=291$, 303; ICD9=291, 303, 305A; ICD-10=F10, O354, Z502, Z714, Z721), alcoholic liver cirrhosis (Swedish revised ICD-7 $=581.10$, 290.10; ICD-8=571.00; ICD-9=571C，571D；ICD-10=K70), and acute and chronic pancreatitis (Swedish revised ICD$7=587 ;$ ICD-8=577; ICD-9=577; ICD- $10=$ K85, K86), diagnosed before, at entry, or during follow up, and used these variables as indicators for excess alcohol intake. Stratified analyses were performed on the presence or absence of alcohol related comorbid diseases. We also stratified the analyses by selected cohort characteristics that may influence the results, including follow up duration and age at cohort entry. The $\chi^{2}$ test for linear trends was used to evaluate dose-response relations with duration of follow up and age at entry. ${ }^{14}$

\section{RESULTS}

Selected characteristics of the pernicious anaemia cohort are listed in table 1 . The male:female ratio among patients hospitalised for pernicious anaemia was 1:1.5. Mean age at index hospitalisation was approximately 74 years. The cohort was followed for an average of 7.1 years, yielding a cumulative 161672 person years. There were $2.4 \%, 0.4 \%$, and $2.0 \%$ of patients who had ever been hospitalised for alcoholism, alcoholic liver cirrhosis, or pancreatitis, respectively. In total, 911 $(4.3 \%)$ patients had ever been hospitalised for any of these three comorbid diseases.

Compared with the general population, significant excess risks for squamous cell carcinoma of the oesophagus and stomach cancer distal to the cardia were observed in pernicious anaemia patients (SIR 3.3 (95\% CI 2.4-4.4); SIR 2.4 (95\% CI 2.1-2.7), respectively). In contrast, we did not observe any important excess risks for oesophageal adenocarcinoma or gastric cardia cancer (SIR 1.7 (95\% CI $0.7-3.4$ ); SIR 1.2 (95\% CI $0.6-2.0)$, respectively). No obvious sex differences were noted for any of the four types of cancer under study (table 2).

Table 3 lists the SIR values for non-cardia stomach cancer risk in pernicious anaemia patients. There was no obvious tendency of increasing excess risk with increasing follow up duration. The excess risk decreased with increasing age at index hospitalisation ( $\mathrm{p}$ value for trend $<0.01$ ). SIRs did not vary importantly when stratified by calendar period at cohort entry (data not shown). Analyses limited to patients without alcohol related diseases yielded almost identical results (table 3). We also stratified distal stomach cancer by histology into adenocarcinoma and carcinoids. An increased relative risk for carcinoids was found compared with the general population $(\mathrm{n}=15$, SIR 26.4 (95\% CI 14.8-43.5)) while for adenocarcinoma the increased relative risk was relatively moderate $(\mathrm{n}=177$, SIR $2.2(95 \%$ CI 1.9-2.6)).

Stratified analysis by follow up duration revealed an increasing excess risk for oesophageal squamous cell carcinoma with increasing follow up duration after index hospitalisation ( $\mathrm{p}$ value for trend $=0.03$ ) (table 4 ). The excess risk decreased with increasing age at index hospitalisation $(p$ value for trend $=0.01$ ). No obvious difference in excess risk was observed by calendar period at cohort entry (data not shown). The excess risk pattern was similar when analyses were restricted to patients without any reported alcohol

Table 2 Standardised incidence ratios (SIRs) and 95\% confidence intervals (Cls) for cancers of the oesophagus and stomach among patients hospitalised for pernicious anaemia

\begin{tabular}{|c|c|c|c|c|c|c|c|c|c|c|c|c|}
\hline & \multicolumn{6}{|c|}{ Oesophagus } & \multicolumn{6}{|c|}{ Stomach } \\
\hline & \multicolumn{3}{|c|}{ Squamous cell carcinoma } & \multicolumn{3}{|c|}{ Adenocarcinoma } & \multicolumn{3}{|c|}{ Non-cardia cancer } & \multicolumn{3}{|c|}{ Cardia cancer } \\
\hline & Obs* & SIR & $95 \% \mathrm{Cl}$ & Obs* & SIR & $95 \% \mathrm{Cl}$ & Obs* & SIR & $95 \% \mathrm{Cl}$ & Obs* & SIR & $95 \% \mathrm{Cl}$ \\
\hline $\begin{array}{l}\text { Total } \\
\text { Sex }\end{array}$ & 43 & 3.3 & $2.4-4.4$ & 7 & 1.7 & $0.7-3.4$ & 218 & 2.4 & $2.1-2.7$ & 12 & 1.2 & $0.6-2.0$ \\
\hline Male & 24 & 3.2 & $2.1-4.8$ & 4 & 1.4 & $0.4-3.5$ & 106 & 2.2 & $1.8-2.6$ & 5 & 0.7 & $0.2-1.7$ \\
\hline Female & 19 & 3.3 & $2.0-5.2$ & 3 & 2.4 & $0.5-6.9$ & 112 & 2.6 & $2.1-3.1$ & 7 & 2.0 & $0.8-4.2$ \\
\hline
\end{tabular}


Table 3 Standardised incidence ratios (SIRs) and 95\% confidence intervals (Cls) for cancer of the stomach distal to the cardia among pernicious anaemia patients, stratified by follow up duration and age at index hospitalisation

\begin{tabular}{|c|c|c|c|c|c|c|}
\hline \multirow[b]{2}{*}{ Characteristic } & \multicolumn{3}{|l|}{ Total } & \multicolumn{3}{|c|}{ Without alcohol related diseases } \\
\hline & Obs* & SIR & $95 \% \mathrm{Cl}$ & Obs* & SIR & $95 \% \mathrm{Cl}$ \\
\hline \multicolumn{7}{|l|}{ Follow up (y) } \\
\hline $1-4$ & 115 & 2.4 & $2.0-2.8$ & 113 & 2.4 & $2.0-2.9$ \\
\hline $5-9$ & 57 & 2.1 & $1.6-2.7$ & 55 & 2.1 & $1.6-2.7$ \\
\hline$\geqslant 10$ & 46 & 2.8 & $2.0-3.7$ & 45 & 2.9 & $2.1-3.8$ \\
\hline$p$ value for trend & & 0.56 & & & 0.57 & \\
\hline \multicolumn{7}{|c|}{ Age at index hospitalisation } \\
\hline$<50$ & 14 & 14.6 & $8.0-24.5$ & 14 & 15.7 & $8.6-26.3$ \\
\hline $50-69$ & 69 & 2.9 & $2.3-3.7$ & 66 & 3.0 & $2.3-3.8$ \\
\hline$\geqslant 70$ & 135 & 2.0 & $1.7-2.4$ & 133 & 2.0 & $1.7-2.4$ \\
\hline$p$ value for trend & & $<0.01$ & & & $<0.01$ & \\
\hline
\end{tabular}

Table 4 Standardised incidence ratios (SIRs) and 95\% confidence intervals (Cls) for squamous cell carcinoma of the oesophagus among pernicious anaemia patients, stratified by follow up duration and age at index hospitalisation

\begin{tabular}{|c|c|c|c|c|c|c|}
\hline \multirow[b]{2}{*}{ Characteristic } & \multicolumn{3}{|l|}{ Total } & \multicolumn{3}{|c|}{ Without alcohol related diseases } \\
\hline & Obs* & SIR & $95 \% \mathrm{Cl}$ & Obs* & SIR & $95 \% \mathrm{Cl}$ \\
\hline \multicolumn{7}{|l|}{ Follow up (y) } \\
\hline $1-4$ & 14 & 2.2 & $1.2-3.7$ & 14 & 2.3 & $1.3-3.9$ \\
\hline $5-9$ & 15 & 3.8 & $2.1-6.3$ & 13 & 3.5 & $1.8-5.9$ \\
\hline$\geqslant 10$ & 14 & 4.9 & $2.7-8.2$ & 14 & 5.1 & $2.8-8.6$ \\
\hline$p$ value for trend & & 0.03 & & & 0.03 & \\
\hline \multicolumn{7}{|c|}{ Age at index hospitalisation } \\
\hline$<50$ & 3 & 10.4 & $2.2-30.4$ & 3 & 11.3 & $2.3-33.0$ \\
\hline $50-69$ & 20 & 4.4 & $2.7-6.9$ & 18 & 4.3 & $2.6-6.8$ \\
\hline$\geqslant 70$ & 20 & 2.4 & $1.5-3.7$ & 20 & 2.5 & $1.5-3.8$ \\
\hline$p$ value for trend & & 0.01 & & & 0.01 & \\
\hline
\end{tabular}

*Observed number of cancer cases

tPerson years before the onset of comorbid diseases were allocated to the comorbidity negative stratum.

related comorbidity (alcoholism, alcoholic liver cirrhosis, or pancreatitis) (table 4). Furthermore, excluding patients with any alcohol relative lesions-for example, those who were hospitalised for trauma twice or more, or those who had any psychiatric diseases-did not change the risk pattern materially (data not shown).

\section{DISCUSSION}

In this large cohort study, we confirmed the previously known strong association between pernicious anaemia and the risk of stomach cancer. Here, we demonstrated that this association was confined to the distal part of the stomach. We also observed an increased risk of oesophageal squamous cell carcinoma whereas the risk of oesophageal adenocarcinoma was only moderately and non-significantly increased.

The strengths of our study include the cohort study design and virtually complete follow up for up to 30 years. The largest sample size to date enabled us for the first time to study the rare outcomes in pernicious anaemia patients. The limitations of our study, as commonly encountered in register based studies, included first and foremost the lack of information on potential confounders, such as alcohol. However, excluding alcohol related diseases did not change the results materially, indicating that confounding by alcohol is unlikely to be a major explanation for the observed obvious excess risk of oesophageal squamous cell carcinoma. In contrast, the decreased SIR for lung cancer in pernicious anaemia patients (SIR $0.8(95 \%$ CI 0.6-1.0)), which is strongly linked to smoking, suggested that smokers were underrepresented in this cohort. Also, as smoking is strongly linked to alcoholism, the lung cancer data indicate indirectly that the prevalence of alcoholism in our cohort was no greater than in the general population that served as a reference.

In our study, we confirmed the elevated risk of oesophageal cancer in pernicious anaemia patients in a previous report. ${ }^{3}$ Furthermore, we found that this increased risk was confined mainly to oesophageal squamous cell carcinoma. Confounding may not explain the observed association and vitamin B12 deficiency has also not been found to be a risk factor for oesophageal squamous cell carcinoma. In pernicious anaemia patients, achlorhydria may exist a long time before a clinical diagnosis is made, which may constitute an intragastric milieu favouring bacterial overgrowth and bacterial n-nitrosation. ${ }^{15}$ $\mathrm{N}$-nitrosamines are considered an important risk factor for oesophageal squamous cell carcinoma. ${ }^{16}$ The fact that in animal experiments oesophageal cancer can be induced by $\mathrm{N}$-nitroso compounds by any route of administration raises the possibility of an important role of endogenous nitrosamines in the carcinogenesis of oesophageal squamous cell carcinoma as nitrosamines generated intragastrically can reach the oesophageal mucosa not only through reflux but also through submucosal veins from the stomach. This hypothesis is partly supported by an ecological study in China in which mortality from oesophageal cancer in 69 counties was significantly correlated with endogenous nitrosation ability, measured by the $\mathrm{N}$-nitrosoproline test. ${ }^{17}$ In this study, $\mathrm{N}$-nitrosoproline formation was significantly reduced by administration of ascorbic acid which may partly explain findings in many epidemiological studies in which ascorbic 
acid and fresh vegetable/fruits have been found to have protective effects against oesophageal squamous cell carcinoma.$^{18}$ Further studies are clearly needed to study intragastric formation, transportation, and metabolism of nitrosamines, and their role in the carcinogenesis of oesophageal squamous cell carcinoma.

Although based on a small number of cases, we found no obviously decreased risk of oesophageal or cardia adenocarcinoma in patients with long term achlorhydria. This finding indicates that gastric acid may not be an essential factor in the carcinogenesis of these cancers. Although the role of acid in the pathogenesis of gastro-oesophageal disease has been well established, acid per se can cause oesophageal mucosal damage only at a very low $\mathrm{pH}$. In acid reflux contents, pepsin seems to play a more important role in causing oesophagitis. ${ }^{19}$ In the acid absent environment, reflux of duodenal contents may even cause more severe oesophagitis as acid can inactivate trypsin, and soluble bile salt can potentiate the activity of pepsin in an alkaline environment. ${ }^{19}$ Duodenal contents have also been shown to be a carcinogen for oesophageal adenocarcinoma in animal models. ${ }^{20}$

In summary, type A atrophic gastritis is associated with an increased risk of distal stomach cancer, and for squamous cell carcinoma of the oesophagus. In contrast, we did not observe a decreased risk of oesophageal adenocarcinoma or cardia cancer in these patients, suggesting that acid reflux may not be a critical factor in the carcinogenesis of these cancers.

\section{ACKNOWLEDGEMENT}

This study was supported by grant 4279-B99-01XAB from the Swedish Cancer Society.

\section{Authors' affiliations}

W Ye, O Nyrén, Department of Medical Epidemiology, Karolinska Institutet, Stockholm, Sweden

\section{REFERENCES}

1 Toh BH, van Driel IR, Gleeson PA. Pernicious anemia. N Engl J Med 1997;337: 1441-8.
2 Brinton LA, Gridley G, Hrubec Z, et al. Cancer risk following pernicious anaemia. Br J Cancer 1989:59:810-3.

3 Hsing AW, Hansson LE, McLaughlin JK, et al. Pernicious anemia and subsequent cancer. A population-based cohort study. Cancer 1993;71:745-50.

4 Mellemkjaer L, Gridley $G$, Moller $H$, et al. Pernicious anaemia and cancer risk in Denmark. Br J Cancer 1996;73:998-1000.

5 Schafer LW, Larson DE, Melton Ld, et al. Risk of development of gastric carcinoma in patients with pernicious anemia: a population-based study in Rochester, Minnesota. Mayo Clin Proc 1985;60:444-8.

6 Blot WJ, McLaughlin JK. The changing epidemiology of esophageal cancer. Semin Oncol 1999;26(suppl 15):2-8.

7 Farrow DC, Vaughan TL, Sweeney C, et al. Gastroesophageal reflux disease, use of $\mathrm{H} 2$ receptor antagonists, and risk of esophageal and gastric cancer. Cancer Causes Control 2000:11:231-8.

8 Chow WH, Finkle WD, Mclaughlin JK, et al. The relation of gastroesophageal reflux disease and its treatment to adenocarcinomas of the esophagus and gastric cardia. JAMA 1995;274:474-7.

9 Lagergren J, Bergstrom R, Lindgren A, et al. Symptomatic gastroesophageal reflux as a risk factor for esophageal adenocarcinoma. N Engl J Med 1999:340:825-31.

10 Mirvish SS. Studies on experimental animals involving surgical procedures and/or nitrosamine treatment related to the etiology of esophageal adenocarcinoma. Cancer Lett 1997;117:161-74.

11 Chow WH, Blaser M, Blot WJ, et al. An inverse relation between $\operatorname{cagA}+$ strains of Helicobacter pylori infection and risk of esophageal and gastric cardia adenocarcinoma. Cancer Res 1998;58:588-90.

12 Nyren O, McLaughlin JK, Gridley G, et al. Cancer risk after hip replacement with metal implants: a population- based cohort study in Sweden. J Natl Cancer Inst 1995;87:28-33.

13 Mattsson B, Rutqvist LE, Wallgren A. Undernotification of diagnosed cancer cases to the Stockholm Cancer Registry. Int J Epidemiol 1985;14:64-9.

14 Breslow NE, Day NE. The design and analysis of cohort studies. In: Statistical Methods in Cancer Research, vol 2. Lyon: International Agency for Research on Cancer, 1987; IARC Scientific Publications No 82.

15 Yeomans ND, Brimblecombe RW, Elder J, et al. Effects of acid suppression on microbial flora of upper gut. Dig Dis Sci 1995;40(suppl):81-95S

16 Craddock VM. Aetiology of oesophageal cancer: some operative factors. Eur J Cancer Prev 1992;1:89-103.

17 Wu Y, Chen J, Ohshima H, et al. Geographic association between urinary excretion of $\mathrm{N}$-nitroso compounds and oesophageal cancer mortality in China. Int J Cancer 1993;54:713-9.

18 Munoz N, Day NE. Esophageal cancer. In: Schotenfeld D, Fraumeni JFJ, eds. Cancer Epidemiology and Prevention. New York: Oxford University Press, 1996:681-706.

19 Peters JH, DeMeester TR. Gastroesophageal reflux. Surg Clin North Am 1993;73: $1119-44$

20 Byrne JP, Attwood SE. Duodenogastric reflux and cancer. Hepatogastroenterology 1999;46:74-85. 